\title{
AIF1 Gene RS2269475:C>T Polymorphism is Associated With Rheumatoid Arthritis Development but not With Disease Activity
}

\author{
Andrzej PAWLIK, ${ }^{1}$ Violetta DZIEDZIEJKO, ${ }^{2}$ Mateusz KURZAWSKI, ${ }^{3}$ Krzysztof SAFRANOW ${ }^{2}$ \\ ${ }^{1}$ Department of Pharmacokinetics and Therapeutic Drug Monitoring, Pomeranian Medical University, Szczecin, Poland \\ ${ }^{2}$ Department of Biochemistry and Medical Chemistry, Pomeranian Medical University, Szczecin, Poland \\ ${ }^{3}$ Department of Experimental \& Clinical Pharmacology, Pomeranian Medical University, Szczecin, Poland
}

\begin{abstract}
Objectives: This study aims to examine whether the allograft inflammatory factor-1 (AlF1) gene rs2269475:C>T polymorphism is associated with rheumatoid arthritis (RA) susceptibility, and whether this polymorphism influences disease activity in patients with RA.

Patients and methods: We examined 380 patients $(62$ males, 318 females; mean age $58.1 \pm 12.9$ years; range 20 to 78 years; age at disease onset $47.4 \pm 13.5$ years) with RA diagnosed according to the criteria of American College of Rheumatology and 378 healthy control subjects (126 males, 252 females; mean age $69.6 \pm 12.1$ years; range 21 to 75 years). Disease activity score in 28 joints (DAS28) was calculated. The active form of RA was diagnosed when DAS28 was $>2.5$, the non-active form was diagnosed when DAS28 was $\leq 2.5$.

Results: The frequency of TT genotype was significantly higher in RA patients compared with the controls (TT vs. CT+CC: odds ratio=6.22, 95\% confidence interval: $1.82-21.28 ; p=0.0013$ ). The frequency of T allele was $18.55 \%$ among RA patients and $12.04 \%$ among healthy subjects (odds ratio $=1.67,95 \%$ confidence interval $=1.25-2.21 ; p=0.00046)$. There were no statistically significant differences in distribution of genotypes and alleles between patients with active and non-active disease (DAS28 $>2.5$ and $\leq 2.5$, respectively).

Conclusion: The results of present study suggest that AIF1 gene rs2269475 polymorphism is associated with RA development; however, has no influence on disease activity.

Keywords: Allograft inflammatory factor-1; disease activity score in 28 joints; polymorphism; rheumatoid arthritis.
\end{abstract}

Rheumatoid arthritis (RA) is a multifactorial disease in which genetic and immunologic factors play an important role in pathogenesis. Allograft inflammatory factor-1 (AIF-1) is the 143-amino acid cytokine primarily identified in rat cardiac allografts with chronic rejection. ${ }^{1}$ AIF-1 is involved in the inflammatory response associated with transplant rejection and has documented expression in various human tissues and cells, such as macrophages and peripheral blood leukocytes. ${ }^{2}$ AIF-1 is produced by macrophages and lymphocytes and its synthesis is mediated by several cytokines such as interferon gamma. ${ }^{3}$ AIF-1 plays an important role in inflammatory responses and the immune activation and function of macrophages. The consequences of an immune response depend on cytokines and other molecules and mediators which are produced and reflect properties of activated T-cells, particularly their differentiation state. ${ }^{4}$ In the pathogenesis of RA, numerous proinflammatory cytokines and other mediators enhancing inflammatory response are involved. ${ }^{5}$ Kimura et al. ${ }^{6}$ suggest that AIF-1 is the cytokine playing the significant role in RA pathogenesis.

Several single nucleotide polymorphisms have been identified in the AIF1 gene., ${ }^{7,8}$ In our previous study, we indicated that AIF1 gene rs2269475:C>T polymorphism is associated 
with increased risk of RA development. ${ }^{9}$ The rs2269475:C $>\mathrm{T}$ polymorphism determines nonsynonymous change from arginine to tryptophan at position 15 of polypeptide. In this study, we aimed to examine whether AIF1 gene rs2269475:C>T polymorphism is associated with RA susceptibility, and whether this polymorphism influences disease activity in patients with RA.

\section{PATIENTS AND METHODS}

We examined 380 patients (62 males, 318 females; mean age $58.1 \pm 12.9$ years; range 20 to 78 years; age at disease onset $47.4 \pm 13.5$ years) with RA diagnosed according to the criteria of American College of Rheumatology. Patients were recruited from the outpatient and inpatient population of the Department of Rheumatology. All subjects were Caucasian from the Pomeranian region of Poland. The subjects enrolled in the study underwent routine biochemical blood analysis, and when required, assays for anticardiolipin antibodies, antinuclear, and immunological complexes. X-rays of the chest, hands, and feet were obtained in all patients and radiographs of other joints were performed when required. These were interpreted by two expert radiologists. The evaluation of the subjects included physical examination, with particular focus on the pattern of joint involvement and the occurrence of extra-articular features (such as vasculitis, anemia, sicca syndrome, amyloidosis, organ involvement), and laboratory features, such as rheumatoid factor. Disease activity score in 28 joints (DAS28) was calculated. ${ }^{10}$ The active form of RA was diagnosed when DAS28 was $>2.5$, the non-active form was diagnosed when DAS28 was $\leq 2.5 .{ }^{11}$
The control group was selected randomly from the population of Pomeranian region of Poland and consisted of 378 Caucasian healthy subjects (126 males, 252 females; mean age 69.6 \pm 12.1 years; range 21 to 75 years). The study was approved by the local ethics committee and written informed consent was obtained from all subjects.

Genomic DNA was extracted from $200 \mu \mathrm{L}$ of whole blood samples using GeneMATRIX Quick Blood DNA Purification Kit (EURx, Ltd. Gdansk, Poland). Single nucleotide polymorphism within the AIF1 gene was genotyped using Taqman realtime polymerase chain reaction genotyping assay (Real-Time polymerase chain reaction; Applied Biosystems, USA) per manufacturer's instruction.

\section{Statistical analyses}

Chi-squared or Fisher's exact tests were used. Odds ratios and its 95\% confidence intervals were calculated. $P$ value of $<0.05$ was considered statistically significant.

\section{RESULTS}

Genotypes of the AIF1 polymorphisms were in Hardy-Weinberg equilibrium ( $p>0.05)$ both in the $\mathrm{RA}$ and control groups.

The distributions of studied genotypes are presented in Table 1 . The frequency of TT genotype was significantly higher in RA patients compared with the controls (TT vs. CT+CC: odds ratio $=6.22$, 95\% confidence interval: 1.82 21.28; $\mathrm{p}=0.0013)$. The frequency of $\mathrm{T}$ allele was $18.55 \%$ among RA patients and $12.04 \%$ among healthy subjects (odds ratio $=1.67,95 \%$ confidence interval=1.25-2.21; $p=0.00046)$. The frequency of $\mathrm{T}$ allele in the controls (12.04\%) was similar to

Table 1. Allograft inflammatory factor-1 rs2269475 genotypes in rheumatoid arthritis patients and controls

\begin{tabular}{|c|c|c|c|c|c|c|c|c|}
\hline & \multicolumn{2}{|c|}{ RA patients $(n=380)$} & \multicolumn{2}{|c|}{ Control group $(n=378)$} & \multirow[t]{2}{*}{$p^{*}$} & \multirow[t]{2}{*}{ Comparison } & \multirow[t]{2}{*}{$p^{* *}$} & \multirow[t]{2}{*}{ OR $(95 \%$ CI $)$} \\
\hline & $\mathrm{n}$ & $\%$ & $\mathrm{n}$ & $\%$ & & & & \\
\hline \multicolumn{9}{|c|}{ AIF1 rs2269475 genotype } \\
\hline $\mathrm{CC}$ & 257 & 67.63 & 290 & 76.72 & 0.0006 & TT+CT vs. CC & 0.0058 & $1.58(1.14-2.17)$ \\
\hline CT & 105 & 27.63 & 85 & 22.49 & & TT vs. $\mathrm{CT}+\mathrm{CC}$ & 0.0013 & $6.22(1.82-21.28)$ \\
\hline TT & 18 & 4.74 & 3 & 0.79 & & TT vs. CC & 0.0005 & $6.77(1.97-23.25)$ \\
\hline \multicolumn{9}{|c|}{ AIF1 rs2269475 allele } \\
\hline C & 619 & 81.45 & 665 & 87.96 & & & & \\
\hline $\mathrm{T}$ & 141 & 18.55 & 91 & 12.04 & & T vs. $C$ & 0.00046 & $1.67(1.25-2.21)$ \\
\hline
\end{tabular}




\begin{tabular}{|c|c|c|c|c|c|c|c|c|}
\hline & \multicolumn{2}{|c|}{$\begin{array}{l}\text { Non-active RA } \\
\text { (DAS28 } \leq 2.5 \text { ) }\end{array}$} & \multicolumn{2}{|c|}{$\begin{array}{c}\text { Active RA } \\
\text { (DAS28 }>2.5 \text { ) }\end{array}$} & \multirow[t]{2}{*}{$p^{*}$} & \multirow[t]{2}{*}{ Comparison } & \multirow[t]{2}{*}{$p^{* * *}$} & \multirow[t]{2}{*}{ OR $(95 \%$ CI $)$} \\
\hline & $\mathrm{n}$ & $\%$ & $\mathrm{n}$ & $\%$ & & & & \\
\hline \multicolumn{9}{|c|}{ AIF1 rs2269475 genotype } \\
\hline $\mathrm{CC}$ & 115 & 44.75 & 142 & 55.25 & & $\mathrm{TT}+\mathrm{CT}$ vs. $\mathrm{CC}$ & 0.58 & $1.14(0.74-1.75)$ \\
\hline $\mathrm{CT}$ & 49 & 46.67 & 56 & 53.33 & 0.66 & TT vs. $\mathrm{CT}+\mathrm{CC}$ & 0.47 & $1.50(0.58-3.91)$ \\
\hline TT & 10 & 55.56 & 8 & 44.44 & & TT vs. CC & 0.46 & $1.54(0.59-4.04)$ \\
\hline \multicolumn{9}{|c|}{ AIF1 rs2269475 allele } \\
\hline C & 279 & 80.17 & 340 & 82.52 & & & & \\
\hline $\mathrm{T}$ & 69 & 19.83 & 72 & 17.48 & & T vs. $C$ & 0.45 & $1.17(0.81-1.68)$ \\
\hline
\end{tabular}

that observed by Alkassab et al. ${ }^{8}$ in 582 healthy Caucasians (12.2\%). Moreover, we compared the frequency of genotypes and alleles between patients with active and non-active disease (DAS28 $>2.5$ and $\leq 2.5$ ). As shown in Table 2, the differences were not statistically significant.

\section{DISCUSSION}

In this study, we examined whether AIF1 gene rs2269475: C>T polymorphism is associated with RA development and disease activity. Present results confirm our previous results suggesting that rs2269475 $\mathrm{T}$ allele is associated with RA susceptibility; additionally, we have revealed that this allele is not associated with RA activity. In another study, we have shown that the AIF1 rs2259571 CC genotype is associated with active form of RA. ${ }^{7}$ The important role of AIF1 gene polymorphisms in RA pathogenesis was indicated in a study by Harney et al. ${ }^{12}$ which examined the AIF1 gene rs2269475 and rs2259571 polymorphisms in RA patients. These authors have shown a significant association between RA and AIF1 haplotypes independent of human leukocyte antigen-DRB1 (HLA-DRB1). Moreover they have demonstrated increased expression of AIF-1 in mononuclear cells of RA patients compared with healthy controls. The expression of AIF-1 was also significantly increased in synovial macrophages from RA patients. These authors suggest that polymorphisms of AIF1 gene are associated with RA independent of the nearby HLA-DRB1, and AIF-1 is directly involved in RA pathogenesis. ${ }^{12}$ AIF1 and HLA-DRB1 genes are located on chromosome 6 and the distance between them is over $900 \mathrm{~kb}$. Analysis of HapMap Phase III dataset revealed that there is no significant linkage disequilibrium between the studied AIF1 single nucleotide polymorphisms and polymorphisms in HLA-DRB1 region, since most of $r 2$ values were lower than 0.01 and none of them was higher than 0.08 .

Kimura et al. ${ }^{6}$ examined the expression of AIF-1 in synovial tissues and measured AIF-1 in synovial fluid derived from patients with either RA or osteoarthritis as well as assessed the proliferation of synovial cells and induction of interleukin 6 (IL-6) following AIF-1 stimulation. The authors have shown that AIF-1 was strongly expressed in infiltrating mononuclear cells and synovial fibroblasts in RA compared with osteoarthritis. Synovial expression of AIF-1 in RA was significantly greater than the expression in osteoarthritis. AIF-1 induced the proliferation of cultured synovial cells and increased the IL- 6 production of synovial fibroblasts and mononuclear cells. The levels of AIF-1 protein were significantly higher in synovial fluid from RA patients compared with osteoarthritis. Furthermore, the concentration of AIF-1 significantly correlated with the IL-6 concentration, which plays an important role in inflammatory process in RA. The authors suggest that AIF-1 is closely associated with the pathogenesis of RA and is a novel member of the cytokine network involved in the immunological processes underlying RA. ${ }^{6}$

AIF-1 is a cytokine playing the significant role in chronic inflammatory processes, 
especially those involving macrophages. AIF-1 was detected in activated macrophages and lymphocytes. ${ }^{13,14}$ Expression of AIF-1 can affect macrophage functional state. In vitro experiments showed that interferon gamma could up-regulate the expression of AIF-1 in macrophages. ${ }^{15}$ Moreover, it was shown that macrophage cell line expressed a certain level of endogenous AIF-1 which could be enhanced by pro-inflammatory cytokines IL-1 $\beta$ or tumor necrosis factor-alpha. Macrophages stimulated with AIF-1 produced significantly increased amounts of IL- 6 , IL-10 and IL-12. ${ }^{16}$ The release by macrophages inflammatory mediators plays the important role in the pathogenesis of RA and in the maintenance of inflammation in joints. ${ }^{17}$ Aforementioned reports indicate that AIF-1 is the cytokine playing the important role in the immune response which may be involved in the pathogenesis of RA. The results of present study suggest that AIF1 gene rs2269475 polymorphism is associated with RA development; however, has no influence on disease activity. The TT homozygotes have much higher risk of RA than CT heterozygotes. The higher number of rs2269475 $\mathrm{T}$ alleles is associated with higher risk of RA, which suggests additive model of genotype-phenotype association. Nevertheless, the role of AIF-1 and AIF1 gene polymorphism in RA pathogenesis require further examinations.

\section{Declaration of conflicting interests}

The authors declared no conflicts of interest with respect to the authorship and/or publication of this article.

\section{Funding}

The study was sponsored by a grant from Polish Ministry of Science and Higher Education (no. NN 402 266 636).

\section{REFERENCES}

1. Autieri MV, Kelemen S, Thomas BA, Feller ED, Goldman BI, Eisen HJ. Allograft inflammatory factor-1 expression correlates with cardiac rejection and development of cardiac allograft vasculopathy. Circulation 2002;106:2218-23.

2. Deininger MH, Meyermann R, Schluesener HJ. The allograft inflammatory factor-1 family of proteins. FEBS Lett 2002;514:115-21.
3. Yang ZF, Ho DW, Lau CK, Lam CT, Lum $\mathrm{CT}$, Poon RT, et al. Allograft inflammatory factor-1 (AIF-1) is crucial for the survival and pro-inflammatory activity of macrophages. Int Immunol 2005;17:1391-7.

4. Utans U, Quist WC, McManus BM, Wilson JE, Arceci RJ, Wallace AF, et al. Allograft inflammatory factory-1. A cytokine-responsive macrophage molecule expressed in transplanted human hearts. Transplantation 1996;61:1387-92.

5. Lundy SK, Sarkar S, Tesmer LA, Fox DA. Cells of the synovium in rheumatoid arthritis. T lymphocytes. Arthritis Res Ther 2007;9:202.

6. Kimura M, Kawahito Y, Obayashi H, Ohta M, Hara H, Adachi $\mathrm{T}$, et al. A critical role for allograft inflammatory factor- 1 in the pathogenesis of rheumatoid arthritis. J Immunol 2007;178:3316-22.

7. Pawlik A, Kurzawski M, Dziedziejko V, Safranow K, Paczkowska E, Maslinski W, et al. Allograft inflammatory factor- 1 gene polymorphisms in patients with rheumatoid arthritis. Genet Test Mol Biomarkers 2012;16:341-5.

8. Alkassab F, Gourh P, Tan FK, McNearney T, Fischbach M, Ahn C, et al. An allograft inflammatory factor 1 (AIF1) single nucleotide polymorphism (SNP) is associated with anticentromere antibody positive systemic sclerosis. Rheumatology (Oxford) 2007:46:1248-51.

9. Pawlik A, Kurzawski M, Szczepanik T, Dziedziejko V, Safranow K, Borowiec-Chlopek Z, et al. Association of allograft inflammatory factor-1 gene polymorphism with rheumatoid arthritis. Tissue Antigens 2008;72:171-5.

10. Fuchs HA, Brooks RH, Callahan LF, Pincus T. A simplified twenty-eight-joint quantitative articular index in rheumatoid arthritis. Arthritis Rheum 1989;32:531-7.

11. Felson DT, Anderson JJ, Boers M, Bombardier C, Chernoff M, Fried B, et al. The American College of Rheumatology preliminary core set of disease activity measures for rheumatoid arthritis clinical trials. The Committee on Outcome Measures in Rheumatoid Arthritis Clinical Trials. Arthritis Rheum 1993;36:729-40.

12. Harney SM, Vilariño-Güell C, Adamopoulos IE, Sims AM, Lawrence RW, Cardon LR, et al. Fine mapping of the MHC Class III region demonstrates association of AIF1 and rheumatoid arthritis. Rheumatology (Oxford) 2008;47:1761-7.

13. Kelemen SE, Autieri MV. Expression of allograft inflammatory factor-1 in $\mathrm{T}$ lymphocytes: a role in T-lymphocyte activation and proliferative arteriopathies. Am J Pathol 2005;167:619-26.

14. Utans U, Arceci RJ, Yamashita Y, Russell ME. Cloning and characterization of allograft inflammatory factor-1: a novel macrophage factor identified in rat cardiac allografts with chronic rejection. J Clin Invest 1995;95:2954-62. 
15. Sibinga NE, Feinberg MW, Yang $H$, Werner $F$, Jain MK. Macrophage-restricted and interferon gamma-inducible expression of the allograft inflammatory factor-1 gene requires Pu.1. J Biol Chem 2002;277:16202-10.

16. Watano K, Iwabuchi K, Fujii S, Ishimori N, Mitsuhashi S,
Ato $\mathrm{M}$, et al. Allograft inflammatory factor- 1 augments production of interleukin- $6,-10$ and -12 by a mouse macrophage line. Immunology 2001;104:307-16.

17. Kinne RW, Bräuer R, Stuhlmüller B, PalomboKinne E, Burmester GR. Macrophages in rheumatoid arthritis. Arthritis Res 2000;2:189-202. 